\title{
A New Tool in the Examination of Lungs in the Emergency Department: Lung Ultrasound
}

\author{
Togay Evrin', Burak Katipoglu², Lukasz Szarpak², Abdurrahman Yılmaz, Erden Erol Ünlüer ${ }^{5}$ \\ 'Department of Emergency Medicine, Ufuk University School of Medicine, Dr. Rıdvan Ege Training and Research Hospital Ankara, Turkey \\ 2Department of Emergency Medicine, Ankara Training and Research Hospital Ankara, Turkey \\ ${ }^{3}$ Department of Emergency Medicine, Medical University of Warsaw, Warsaw, Poland \\ ${ }^{4}$ Department of Emergency Medicine, Uşak University School of Medicine Training and Research Hospital, Uşak, Turkey \\ ${ }^{5}$ Department of Emergency Medicine, Uşak University School of Medicine, Uşak, Turkey
}

Cite this article as: Evrin T, Katipoğlu B, Szarpak L, Yılmaz A, Ünlüer EE. A New Tool in the Examination of Lungs in the Emergency Department: Lung Ultrasound. Eurasian J Emerg Med 2017; 16: 137-40

\begin{abstract}
Pneumonia is a worldwide major healthcare and economic problem. It has a considerable effect on morbidity and mortality. The use of lung ultrasound (LUS) has gained popularity in intensive care units and emergency departments (EDs). LUS has recently been shown to be highly effective for evaluating pulmonary conditions and has been increasingly recognized as a potentially useful approach for diagnosing community-acquired pneumonia. Here we present the case of a patient with dyspnea due to pneumonia. Pleural effusion and parancimal images were obtained by LUS, and the pleural thickness was determined. We found that LUS is a useful tool for diagnosing pneumonia and detecting pleural effusion in EDs.
\end{abstract}

Keywords: Consolidation, lung ultrasound, pleural effusion, pneumonia

\section{Introduction}

Pneumonia is a worldwide major healthcare and economic problem and has a considerable effect on morbidity and mortality (1). Using lung ultrasound (LUS) has gained popularity in intensive care units and emergency departments (EDs). It has recently been shown to be highly effective in the evaluation of pulmonary conditions and has become increasingly recognized as a potentially useful approach for diagnosing community-acquired pneumonia $(2,3)$.

\section{Case Presentation}

Here we present the case of a patient with dyspnea due to pneumonia. A 47-year-old male was admitted to the ED with fever, chest pain, cough, and dyspnea for 7 days. Respiratory sounds decreased in the left basal area. He underwent coronary artery bypass graft surgery 3 months ago. His body temperature was $37.1^{\circ} \mathrm{C}$, blood pressure was $110 / 70 \mathrm{mmHg}$, and oxygen saturation was $98 \%$. Initial investigations on admission con- firmed leukocytosis $(10,600 / \mu \mathrm{L}, 84 \%$ neutrophils), anemia (hemoglobin: $9.2 \mathrm{~g} / \mathrm{dL}$, hematocrit of $26 \%, C$ reactive protein CRP level of $128.5 \mathrm{mg} / \mathrm{L}$, and slightly elevated liver enzyme and blood urea nitrogen levels (SGOT: 280 U/L, SGPT: 200 U/L, BUN: 55 mg/dL, and Cr: 0.7 mg/dL).There were no ischemic changes on his electrocardiogram. CK, CKMB, and troponin levels were normal. The D-dimer level was in the normal range. Arterial blood gas analysis revealed hypoxemia $\left(\mathrm{pH}: 7.35, \mathrm{paO}_{2}: 65 \mathrm{mmHg} \mathrm{paCO}_{2}\right.$ : $30 \mathrm{mmHg}$ ). His chest X-ray (CXR) showed a pleural effusion in the left hemithorax (Figure 1). Bedside LUS was performed with a MicroMaxx ${ }^{\circledast}$ ultrasound device equipped with a $3.5-\mathrm{MHz}$ phased array probe and a 5.0-10 MHz phased array convex probe (Sonosight ${ }^{\oplus}$ Bio-medical Electronics Co., Bothel WA 98021 USA). Left lower lobe consolidation with air bronchograms and pleural effusion were detected on performing LUS (Figures 2-5). Patient was hospitalized to the chest diseases service The patient began to follow in chest disease clinic. Antibiotic and anti-inflammatory treatments were initiated. A thoracic computed tomography (CT) scan revealed the presence of unilateral consolidations with pleural thickness and a pleural effusion (Figures 6,7 ). These correlated with our LUS findings. 


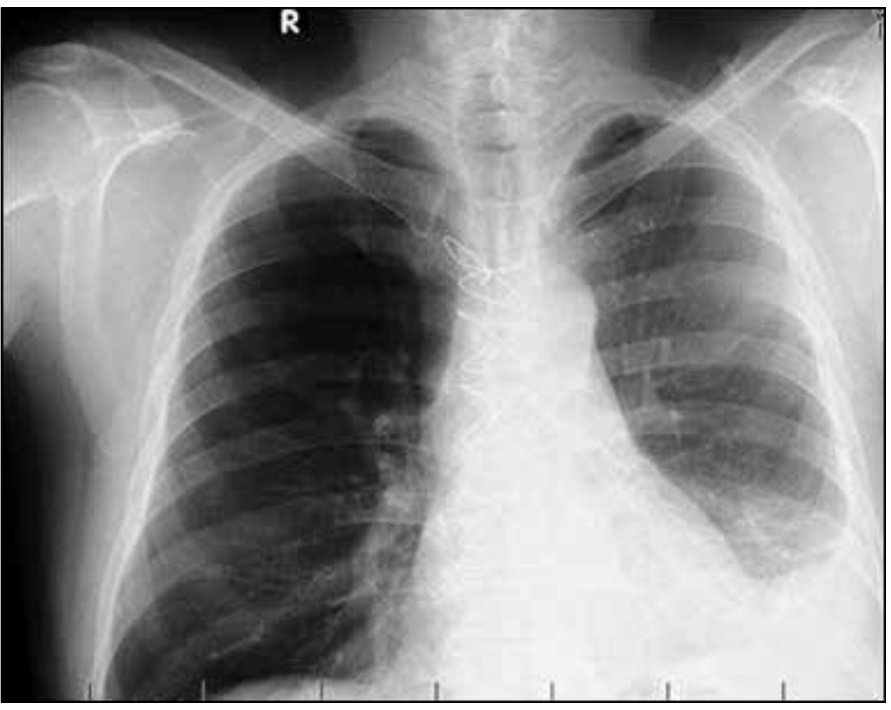

Figure 1. Pleural effusion in the left hemithorax

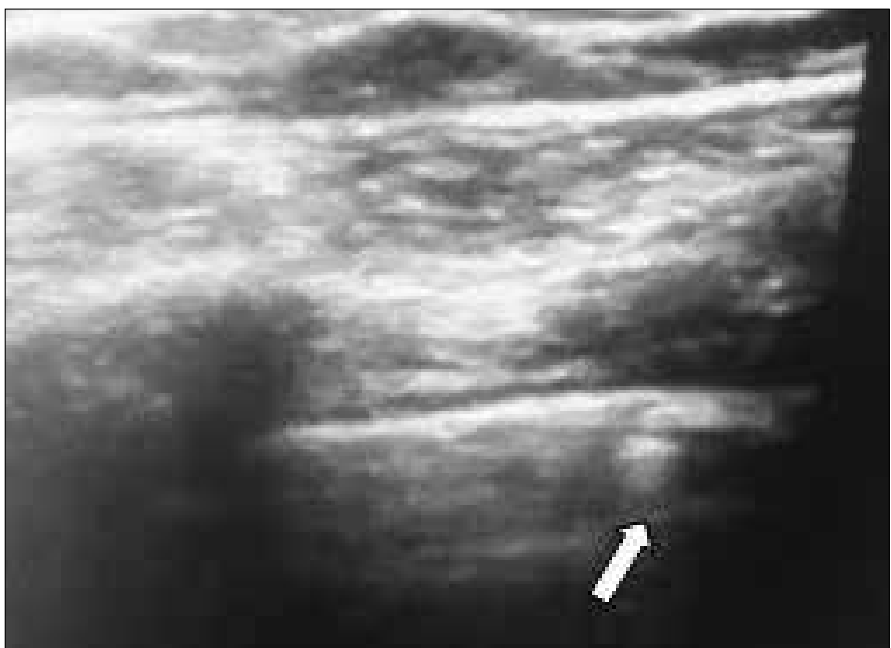

Figure 2. White arrow shows the presence of consolidation with evidence of air bronchograms

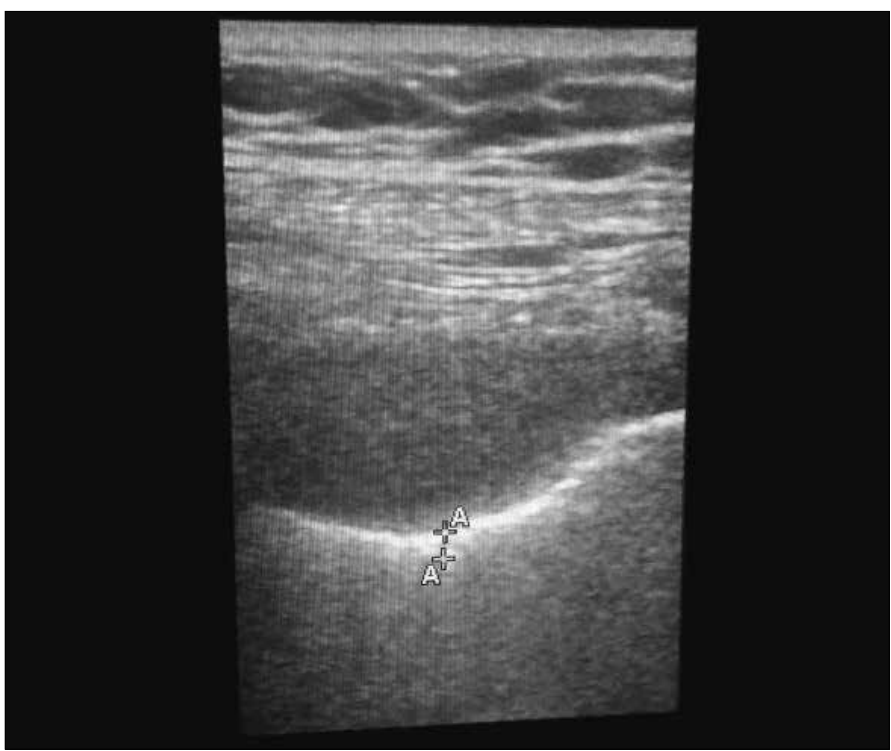

Figure 3. Pleural thickness $(0.23 \mathrm{~cm})$

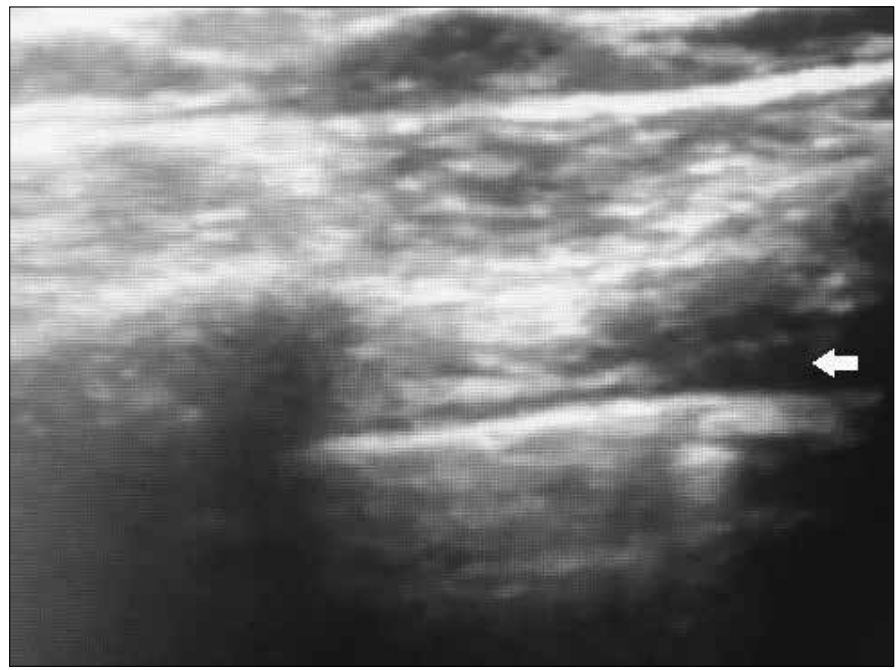

Figure 4. White arrow shows ultrasonographic visualization of a pleural effusion between the visceral and parietal pleura

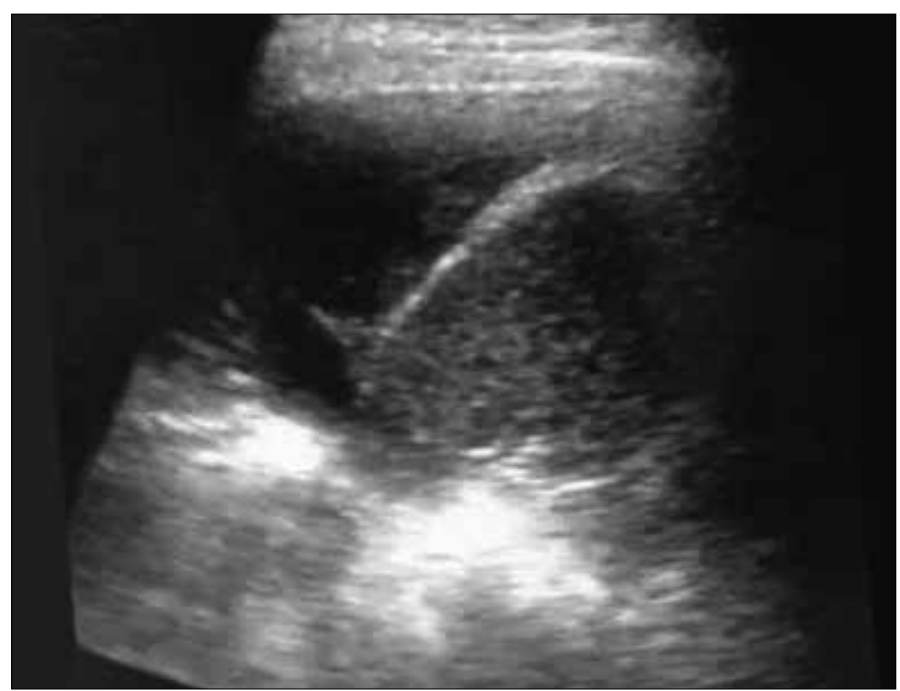

Figure 5. Ultrasonographic visualization of a pleural effusion in the left lung

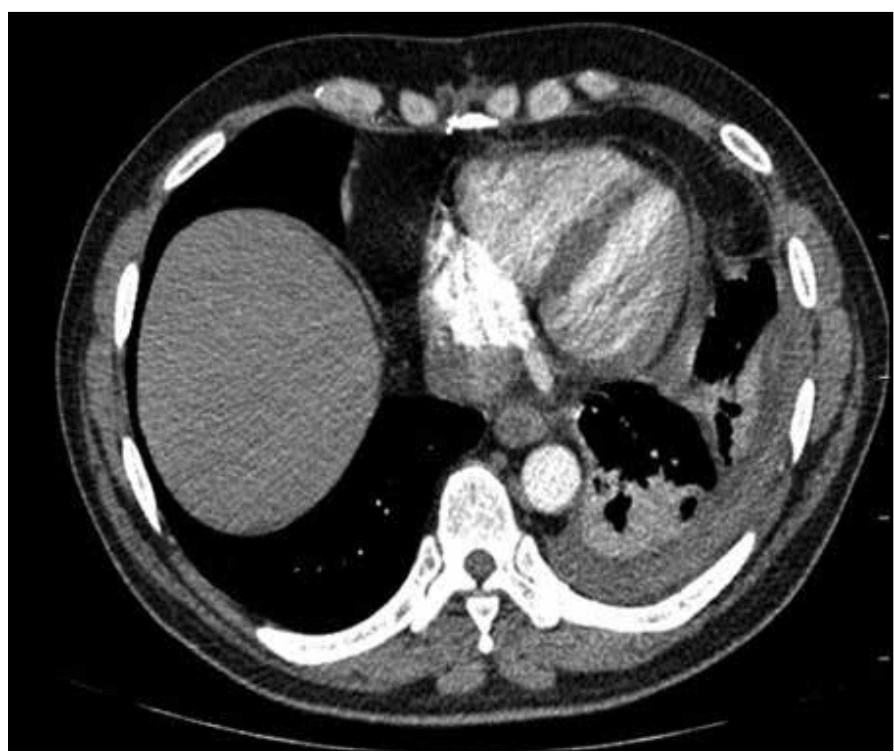

Figure 6. Pleural effusion and thickness, atelectasis 


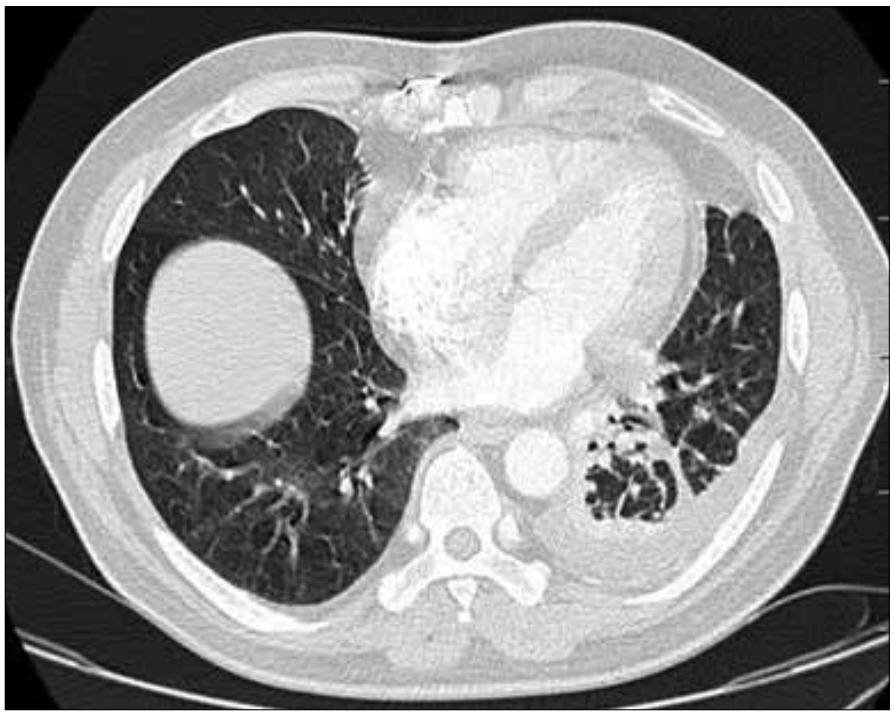

Figure 7. Pleural effusion

\section{Discussion}

Many articles from the literature have shown the usefulness of LUS ultrasound for detecting alveolar consolidations $(4,5)$. LUS has a high sensitivity (94\%) and specificity (96\%) for diagnosing pneumonia in adults. Its role is known well both as a rule-in and rule-out test for pneumonia in adults admitted to EDs and medical wards. Even in patients with acute dyspnea, for which the differential diagnosis may be broad, LUS is a good tool for discrimination. In a meta-analysis, Chavez et al. (6) mentioned that LUS conducted by highly skilled sonographers can be used for diagnosing pneumonia. ED physicians should be encouraged to learn LUS for diagnosing pneumonia because it appears to be an established diagnostic tool in the hands of experienced physicians (6).

The normal pleural thickness is only 0.2 to $0.4 \mathrm{~mm}$. Focal and diffuse pleural thickening, whether due to a tumor or due to inflammation, are exquisitely demonstrated on performing CT, but they are difficult to detect by performing sonography unless the thickness reaches $1 \mathrm{~cm}$ or more. The pleural membranes appear as a single highly echogenic line that moves while breathing (lung sliding) $(7,8)$. In our case, the pleural thickness was $0.23 \mathrm{~cm}$.

The key to the ultrasound visualization of pneumonia in the lungs is the relative loss of the aeration of a portion of the lung and a concomitant increase in the fluid content, which is seen in lung consolidation. Once this consolidation reaches the pleura, it can be seen on performing ultrasound. Although some early consolidation must be localized below the pleura and can be imaged using ultrasound. Current literature suggests that concolidation may be below the pleura (9).

Boundaries of a consolidated lung segment are defined by the pleural line, the adjacent aerated lung, and any effusion that may be present. The boundary created by the adjacent aerated lung would naturally appear irregular. In real time, air can be seen moving through the bronchi, and this finding is known as a dynamic air bronchogram. The sensitivity of B-mode ultrasound imaging is approximately $90 \%$. Consolidation and dynamic air bronchograms have the highest specificity for detecting pneumonia. summarizes the typical ultrasound findings associated with pneumonia (9). According to the study by Lichtenstein et al. (10), LUS can immediately cause acute respiratory failure in $90.5 \%$ of patients in critical care units. Because the air-to-fluid ratio completely differs in different diseases, this results in different artefact patterns visible during LUS. Posterior lung areas can immediately indicate pleural effusion and $90 \%$ of acute alveolar consolidation (AAC) locations. The presence of an air bronchogram and a decrease in lung sliding are other ultrasonographic findings in AAA (11). In our patient, a pleural effusion and an air bronchogram were indicated by LUS (Figures 2, 5). The most common ultrasound findings associated with pneumonia are hypoechoic areas of varying size and shape, irregular and serrated margins, pleural effusions, heterogeneous echo textures, air bronchograms, and dynamic air bronchograms (12). An air bronchogram can be a valuable dynamic sign of alveolar consolidation on performing bedside ultrasonography for diagnosing pneumonia (13).

\section{Conclusion}

Lung ultrasound has some clear advantages over a CXR in patients who are pregnant, bedridden, and in resource-limited settings where CXR machines are not currently available. Moreover, it can be performed at the bedside; the evaluation is easy and fast to perform, and the patient is not exposed to ionizing radiation with LUS. LUS is a valid alternative for diagnosing pneumonia.

Informed Consent: Written informed consent was obtained from patient who participated in this case.

Peer-review: Externally peer-reviewed.

Conflict of Interest: No conflict of interest was declared by the authors.

Financial Disclosure: The authors declared that this study has received no financial support.

\section{References}

1. File TM Jr, Marrie TJ. Burden of community-acquired pneumonia in North American adults. Postgrad Med 2010; 122: 130-41. [CrossRef]

2. Volpicelli G, Elbarbary M, Blaivas M, Lichtenstein DA, Mathis G, Kirkpatrick $A W$, et al. International evidence-based recommendations for pointof-care lung ultrasound. Intensive Care Med 2012; 38: 577-91. [CrossRef]

3. Cortellaro F, Colombo S, Coen D, Duca PG. Lung ultrasound is an accurate diagnostic tool for the diagnosis of pneumonia in the emergency department. Emerg Med J 2012; 29: 19-23. [CrossRef]

4. Mathis G. Thoraxsonography-Part II: Peripheral pulmonary consolidation. Ultrasound Med Biol 1997; 23: 1141-53. [CrossRef]

5. Targhetta R, Chavagneaux R, Bourgeois JM, Dauzat M, Balmes P, Pourcelot L. Sonographic approach to diagnosing pulmonary consolidation. J Ultrasound Med 1992; 11: 667-72. [CrossRef]

6. Chavez MA, Shams N, Ellington LE, Naithani N, Gilman RH, Steinhoff MC, et al. Lung ultrasound for the diagnosis of pneumonia in adults: a systematic review and meta-analysis. Respir Res 2014; 15: 50. [CrossRef]

7. Leung AN, Muller NL, Miller AR. CT in the differential diagnosis of pleural disease. AJR 1990; 154: 487-92. [CrossRef]

8. Lichtenstein DA. Ultrasound in the management of thoracic disease. Crit Care Med 2007; 35(Suppl): S250-61. [CrossRef]

9. Blackmore CC, Black WC, Dallas RV, Crow HC. Pleural fluid volume estimation: a chest radiograph prediction rule. Acad Radiol 1996; 3: 103-9. [CrossRef]

10. Lichtenstein $D$, Mezière GA. Relevance of lung ultrasound in the diagnosis of acute respiratory failure:The BLUE protocol. Chest 2008; 134: 117-25. [CrossRef]

11. Lichtenstein $D$, Mezière $G$, Seitz J. The "aerial dynamic bronchogram", an ultrasound sign of alveolar consolidation. Réanimation 2002; 11(Suppl 3): 98.

12. Blaivas $M$. Lung ultrasound in evaluation of pneumonia. J Ultrasound Med 2012; 31: 823-6. [CrossRef]

13. Ünlüer EE, Karagöz A. A dynamic sign of alveolar consolidation in bedside ultrasonography: Air Bronchogram. Interv Med Appl Sci 2014; 6: 40-2. [CrossRef] 